\title{
Light absorption of organic aerosol from pyrolysis of corn stalk
}

\author{
Xinghua $\mathrm{Li}^{1 *}$, Yanju Chen ${ }^{2}$ and Tami C. Bond ${ }^{2}$ \\ ${ }^{1}$ School of Space and Environment, Beihang University, Beijing 100191, P.R. China \\ ${ }^{2}$ Department of Civil and Environmental Engineering, University of Illinois at Urbana-Champaign, \\ Urbana, IL, 61801, USA
}

\section{ABSTRACT}

Organic aerosol (OA) can absorb solar radiation in the low-visible and ultra-violet wavelengths thereby modifying radiative forcing. Agricultural waste burning emits a large quantity of organic carbon in many developing countries. In this work, we improved the extraction and analysis method developed by Chen and Bond, and extended the spectral range of OC absorption. We examined light absorbing properties of primary OA from pyrolysis of corn stalk, which is a major type of agricultural wastes. Light absorption of bulk liquid extracts of OA was measured using a UV-vis recording spectrophotometer. OA can be extracted by methanol at $95 \%$, close to full extent, and shows polar character. Light absorption of organic aerosol has strong spectral dependence (Absorption Ångström exponent = 7.7) and is not negligible at ultra-violet and low-visible regions. Higher pyrolysis temperature produced OA with higher absorption. Imaginary refractive index of organic aerosol $\left(k_{O A}\right)$ is 0.041 at $400 \mathrm{~nm}$ wavelength and 0.005 at $550 \mathrm{~nm}$ wavelength, respectively. Keywords: organic aerosol, light absorption, corn stalk, pyrolysis, spectral dependence 
Organic aerosol (OA) is ubiquitous, comprising a major fraction (20-90\%) of the atmospheric

fine aerosol mass (Kanakidou et al., 2005; Zhang et al., 2007) around the world, and has important effects on regional and global climate, air quality visibility, and human health. OA mainly scatters incoming solar radiation and many climate models treat it as a purely cooling agent (Chung and modifying radiative forcing (Kirchstetter et al., 2004; Alexander et al., 2008; Chen and Bond, 2010;

Chakrabarty et al., 2010; Lack, et al., 2012; Saleh et al., 2013, 2014). Such OA is referred to as brown carbon (BrC) (Andreae and Gelencser, 2006). A few recent climate modeling studies

Seinfeld, 2002; Ming et al., 2005; Myhre et al., 2007). However, there is a growing body of evidence that OA can absorb radiation in the low-visible (blue) and ultra-violet (UV) wavelengths thereby demonstrated that $\mathrm{BrC}$ was an important atmospheric absorber, globally and regionally (Park et al., 2010; Feng et al., 2013; Lin et al., 2014; Lu et al., 2015). Due to lack of available measurement data on BrC's light absorbing properties, its absorbing effect was simplified in models, which couldn't reflect its absorptive behavior varieties from different sources.

The complex refractive index $(m=n-i k)$ is the fundamental parameter to determine the particle's optical properties. BrC's light absorption is often quantified by mass absorption cross section (MAC, $\mathrm{m}^{2} \mathrm{~g}^{-1}$ ), and its spectral dependence is described by Absorption Ångström Exponent (AAE). MAC can be calculated using Mie theory if $m$ and particle size are known.

Because $\mathrm{BrC}$ is a complex mixture containing a large group of organic compounds with various absorptivities and lacking a formal analytical definition, many methods have been used to determine its light absorbing properties. Kirchstetter et al. (2004) extracted organic carbon (OC) with acetone from the loaded filters and used optical transmission method to determine the difference in the light transmission of the filters before and after extraction, and then estimated optical constants for OC from biomass smoke and motor vehicle. Chen and Bond (2010) extracted OC with different solvents and measured light absorption of bulk liquid extract, and then derived light absorbing properties of 
45 OC from wood pyrolysis. Alexander et al. (2008) applied the electron energy-loss spectrum in the transmission electron microscope and quantified the optical properties of individual amorphous carbon spherical particles in East Asia outflow. Yang et al. (2009) used a multi-wavelength aethalometer to measure atmospheric aerosol light absorption, assumed that black carbon (BC) followed an inverse-wavelength relationship from 370 to $950 \mathrm{~nm}$, BC was the only significant light absorber at 950nm, i.e. light absorption effect of $\mathrm{BrC}$ and dust was negligible at this wavelength, and then derived BrC light absorbing properties. Favez et al. (2009) applied the same method to quantify light absorption of organic aerosol from agricultural waste burning. Several studies employed optical closure methods (Chakrabarty et al., 2010; Lack et al., 2012; Saleh et al., 2013; 2014), namely, the complex refractive index of the particles were retrieved from Mie closure calculation

based on particle size, scattering and absorption measurement. Lu et al. (2015) reviewed available measurements of the light-absorption properties of primary OA from various combustion sources.

Since biofuel combustion and biomass open burning contributes about $90 \%$ of the total primary OC (Bond et al., 2004), investigation of the light absorbing properties of primary OC has been focused on this type of source. However, most of them concern wood combustion, savanna and forest fire, duff combustion (Kirchstetter et al. 2004; Chen and Bond 2010; Chakrabarty et al., 2010; Lack et al., 2012; Saleh et al., 2013; 2014). Agricultural wastes are often burned in the field after harvesting and used as fuel for cooking and heating in developing countries (Li et al, 2007, 2009). These emissions contribute $9 \%$ to global annual OC emission and $27.5 \%$ to its emission in China (Bond et al., 2004; Cao et al., 2006). Only two studies have examined light absorption of OA from burning of rice straw (Favez et al., 2009; Saleh et al., 2014), and it is not known whether OA from waste of other crops, which is structurally different, has similar properties.

In this work, we explored light absorbing properties of primary OA from combustion of corn stalk, which is a major type of agricultural waste. The experiments were designed to generate OA under the pyrolysis combustion phase only, since approximately $80 \%$ of OC is emitted in this 
combustion phase (Einfeld et al., 1991). Roden et al. (2006) used real-time emission measurements

to demonstrate that solid-fuel combustion occurs in distinct phases, with black and brown carbon emitted separately. Real-time measurements (Chen et al., 2011) also show that about half of organic carbon mass is emitted in the rapid ejection that occurs after fuel addition. Therefore, separating combustion by phase, rather than separating particles physically, allows exploration of different realistic aerosol types. with nitrogen $\left(\mathrm{N}_{2}\right)$ as carrier gas, and organic aerosol was generated at three temperatures. We used the improved extraction method and extracted organic aerosol samples with methanol and employed a UV-vis spectrophotometer to measure light absorption of the extracts. We derived absorption per mass of organic aerosol $(\alpha / \rho)$, a parameter related to MAC of OC. We also reported imaginary refractive index of organic aerosol $\left(k_{O A}\right)$ and its absorption Ångström exponent (AAE).

\section{Method} reactor with a flow rate at $6 \mathrm{~L} \mathrm{~min}^{-1}$, which kept the reactor at a slightly positive pressure and

\subsection{Generation and collection of organic aerosol}

Corn stalks were collected from a field, near University of Illinois at Urbana-Champaign (Illinois, USA), after the corn was harvested. The corn stalks were dried in the lab for over a week and then cut into pieces with about $4 \mathrm{~cm}$ in length.

Organic aerosol was generated by pyrolyzing pieces of corn stalks in a temperature-controlled pyrolysis reactor. The reactor consisted of a resistive heating cylinder and a heater with 120 volt, 650 watt (Watlow VC403A06A). A proportional integral differential (PID) controller was used to control the reactor temperature fluctuation due to the pyrolysis process within $10^{\circ} \mathrm{C}$. Corn stalk pieces were placed on a stand, $3 \mathrm{~cm}$ above the bottom center of the pyrolysis reactor. In order to prevent flame and thus the production of $\mathrm{BC}, \mathrm{N}_{2}$ was used as the carrier gas and injected at the bottom of the prevented oxygen from entering. The reactor was previously used to generate organic aerosol from 
wood pyrolysis by Chen and Bond (2010) and Brem et al. (2012). Moreover, before pyrolysis, corn stalk pieces were purged with $\mathrm{N}_{2}$ and vacuumized, and then soaked into $\mathrm{N}_{2}$ for over one day, which removed oxygen within the corn stalk and prevent $\mathrm{BC}$ production during the pyrolysis. In order to investigate the effect of pyrolysis temperature on OC absorption, the experiments were carried out under three temperatures: $210^{\circ} \mathrm{C}, 270{ }^{\circ} \mathrm{C}, 360^{\circ} \mathrm{C}$. These temperatures were chosen, based on biomass thermal degradation behavior (White and Dietenberger, 2001), to span the range of conditions where pyrolysis occurs. In each run, three pieces of corn stalk were pyrolyzed sequentially and the pyrolysis lasted for about 10 minutes. The pyrolysis procedure was designed to simulate the release of organic aerosol from corn stalk combustion under normal combustion temperatures. Pyrolysis product was released from the corn stalk, escaped from the reactor and then formed organic aerosol through partial condensation in the cooling plume.

The aerosol generated by the pyrolysis of corn stalk pieces was sampled $4 \mathrm{~cm}$ above the reactor chimney at a flow rate of $3.75 \mathrm{~L} \mathrm{~min}^{-1}$. The sampling air was then diluted with particle-free dry air with 7:1 dilution ratio. After dilution, the sample temperature was about $30^{\circ} \mathrm{C}$. Before collection, particles larger than $1 \mu \mathrm{m}$ was removed by a two-stage Berner type impactor.

Aerosol was collected by four parallel sampling branches (Fig. 1). The first branch and second branch collected primary OA on 47mm quartz fiber filters (Tissuquartz 2500, Pall Inc.), under high $\left(20 \mathrm{~L} \mathrm{~min}^{-1}\right)$ and low $\left(2 \mathrm{~L} \mathrm{~min}^{-1}\right)$ flow rates, respectively, The purpose of collecting samples at two flow rates will be discussed in Section 2.2. However, quartz filters may also collect adsorbed organic vapors. A Teflon-quartz filter combination has been recommended to estimate this artifact (Turpin et al., 1994), with a front Teflon filter (Millipore, Fluoropore ${ }^{\mathrm{TM}}$ Membrane Filters, FALP04700) removing particles from the gas stream, and a backup quartz filter estimating the gaseous adsorption. The third branch contained this combination. The sampling flow rate in this branch was set to $2 \mathrm{~L}$ $\min ^{-1}$, same as the second branch. The rest of the dilution aerosol flow was bypassed through the fourth branch. 


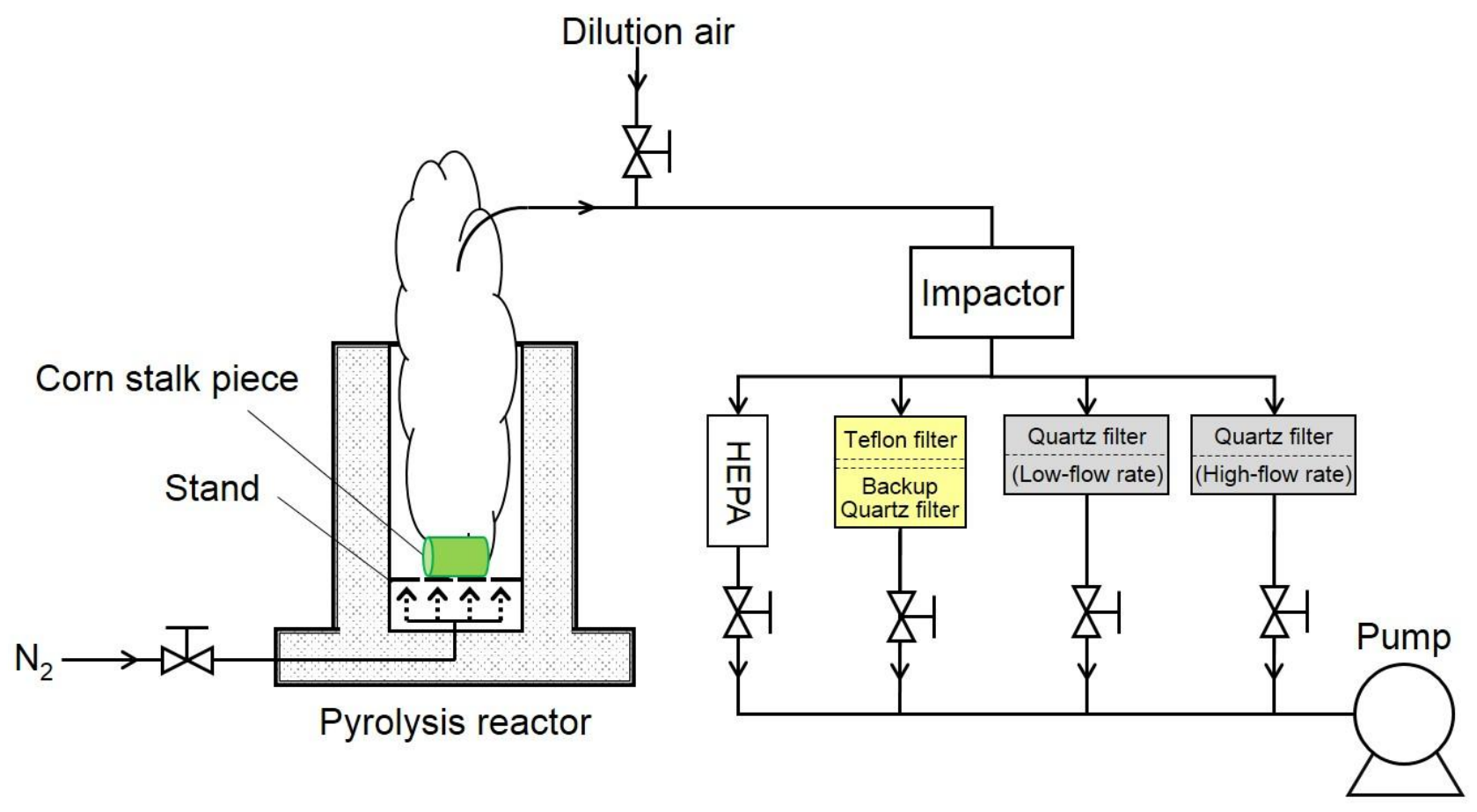

Fig. 1 Schematic of generation and collection system of organic aerosol

2.2 Analysis method

We used the method developed by Chen and Bond (2010) to extract and characterize the spectral light absorption by organic aerosol samples. It involves extracting samples with a solvent, measuring light absorption of the extracts, and determining OC concentrations in the extracts where absorption is measured. However, due to the limit of instrument detection range, the valid range of OC absorption reported in previous study are from $360 \mathrm{~nm}$ and ends around 500nm. Lack of OC absorption at longer wavelength is not conducive to estimate brown carbon total radiative forcing.

To extend the spectral range of OC absorption, the method has been improved by 1) extracting samples both under high and low flow rates, measuring light absorption of the extracts, combining 
136 those two sets of data to obtain valid absorbance values across wavelength spectra ranging from

$137360 \mathrm{~nm}$ to around $600 \mathrm{~nm}$; 2) analyzing OC in samples under the low flow rate directly, measuring

138 OC content in the extracts under the high flow rate by pipetting a droplet of extracts on the blank

139 filter and then analyzing OC after drying, thus determining OC concentrations in the extracts from

140 samples both under high and low flow rate. Analysis procedures of samples collected at three

141 sampling branches are shown in Fig. 2.
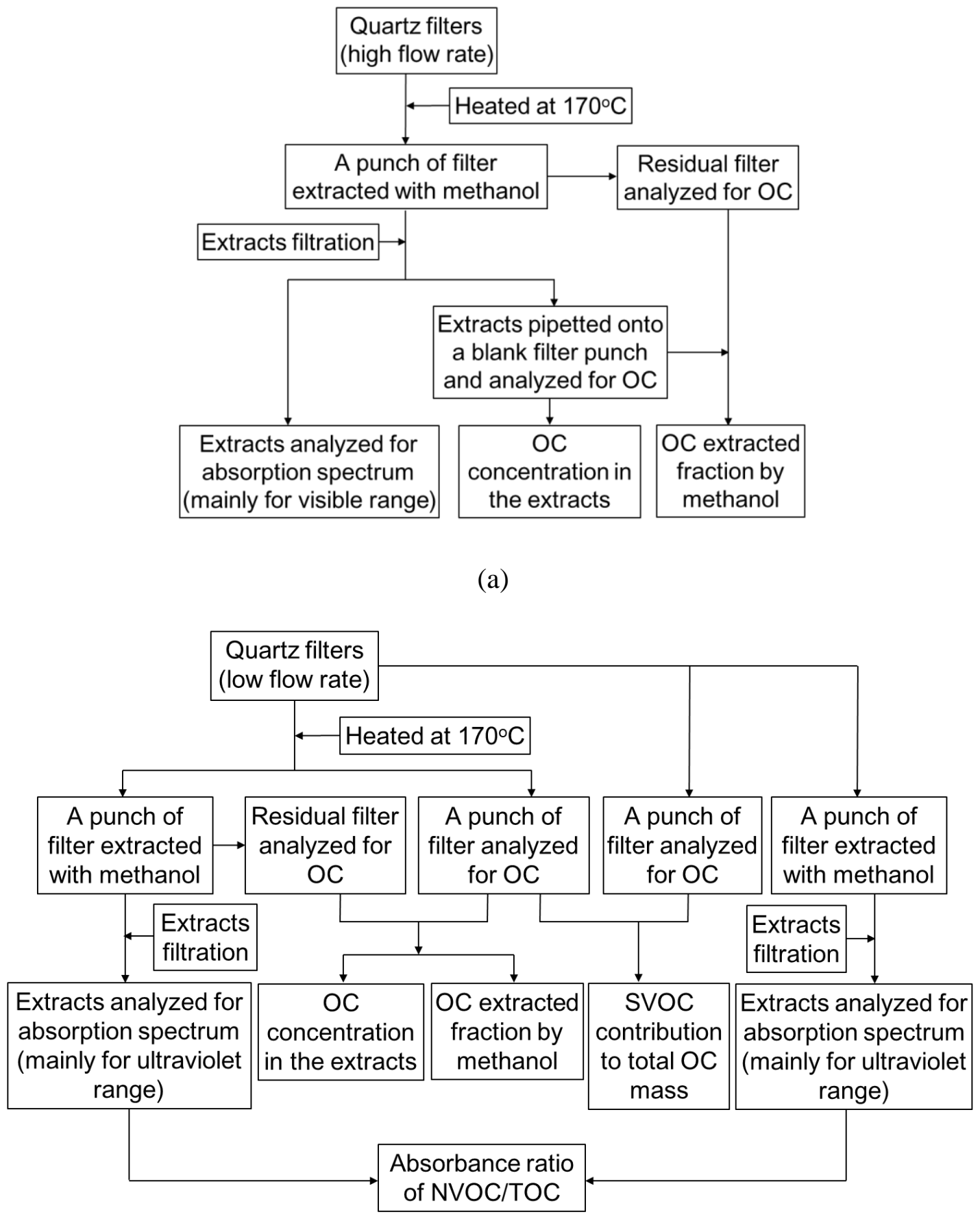


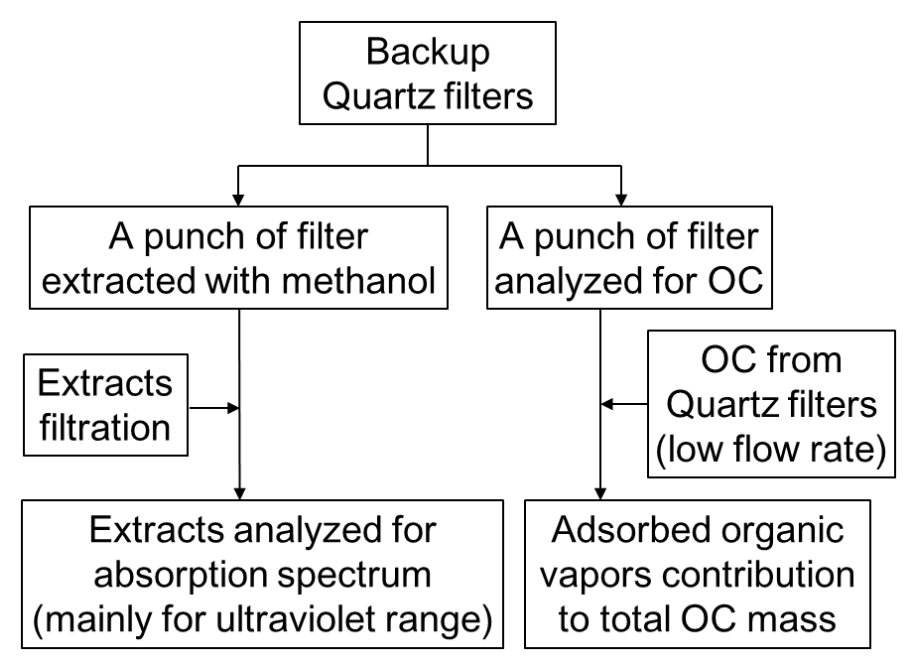

(c)

148 Fig.2 Analysis procedures of samples collected at three sampling branches (a) high flow rate branch,

(b) low flow rate branch, (c) backup filter branch.

\section{$150 \quad$ 2.2.1 Filter extraction}

The loaded quartz filters in the high and low flow rate branches, and backup quartz filters were

152 extracted with methanol (Fisher Scientific, A.C.S. certified), which was found previously to extract 153 organic mass from wood pyrolysis efficiently. Chen and Bond (2010) reported that the fraction of 154 total OC extracted by methanol was from $92 \%$ to $98 \%$. A punch of quartz filter with $3 \mathrm{ml}$ methanol 155 was sonicated for $1 \mathrm{~h}$ in an ultrasonic bath (Model 8892, Cole Parmer, Inc), kept at room temperature 156 for $20 \mathrm{~h}$ to let the solution reach equilibrium, and sonicated for an additional hour. After sonication, 157 all the extracts were filtered by a $25 \mathrm{~mm}$ diameter syringe filter with $0.2 \mu \mathrm{m}$ pore size (Whatman, 158 Anotop@ disposable syringe filter) to remove impurities and undissolved materials for further 159 absorption analysis by UV-vis spectrophotometer. Prior to the filtration, the syringe filter was pre160 washed with $20 \mathrm{ml}$ methanol to remove impurities which could have an effect on further absorption 161 analysis. 
After extraction, residual filters were taken out of the solutions and dried in a fume hood and then kept in dishes immediately and stored in a freezer for post-extraction OC analysis. This analysis 164 determined the amount of unextracted OC.

\subsubsection{Absorbance measurement}

Light absorption of bulk liquid extracts was measured using a UV-vis recording spectrophotometer (Shimadzu, UV-2401) for wavelength spectra ranging from 250nm to 750nm. The instrument was operated in spectrum mode at a wavelength resolution of $1 \mathrm{~nm}$. Each spectrum was determined relative to the extraction solvent, methanol, as reference. According to Chen and Bond (2010), valid absorbance (A) values from the spectrophotometer should be in the range of 0.05 and 1 .

171 The lower limit, $\mathrm{A}=0.05$, is the instrument detection limit. While A values exceed the upper limit, $172 \mathrm{~A}=1$, the Beer-Lambert law is not valid. In previous work (Chen and Bond, 2010), the valid range of 173 OC absorption ended around 500nm, and absorption measured at longer wavelength was usually 174 below the lower limit. In order to meet the requirement, we measured both absorbance of extracts 175 from quartz filters in the high and low flow rate branches. For the former, the absorbance values 176 were often in the valid range in the visible region, but often exceeded the upper limit in the 177 ultraviolet region. Meanwhile, for the latter, its absorbance values were often in the valid range in the 178 ultraviolet region, however often below the lower limit in the visible region. Hence, we combined 179 those two sets of data to obtain valid absorbance values across wavelength spectra ranging from $180360 \mathrm{~nm}$ to around $600 \mathrm{~nm}$.

181 In order to investigate absorbance property of organic vapors adsorbed on the quartz filters, we 182 also measured absorbance of extracts from backup quartz filters.

$183 \quad$ 2.2.3 Determination of OC concentrations in the extracts

OC concentrations in the extracts where absorption is measured were derived by 1) different of 
mass in the volume of extract. OC concentrations in the extracts could not be analyzed by total carbon analyzer because of the organic solvent.

before and after extraction was determined by a thermal-optical OC/EC analyzer (Sunset Laboratory,

For quartz filters in the low flow rate branch and backup quartz filters, OC content on the filters Tigard, OR) using the NIOSH protocol (2003). The choice of temperature protocol can affect the division between OC and EC (Chow et al., 2004), but the aerosol generated in this work was entirely OC, so the protocol used does not affect the findings.

OC loading on the quartz filters in the high flow rate branch before extraction was above the valid range of the OC/EC analyzer. The exact OC content could not be obtained by direct analysis of quartz filters. Instead, $30 \mu$ of extract was pipetted onto a blank $1 \mathrm{~cm}^{2}$ quartz filter punch and then the punch with the extract was dried for 30 minutes in a fume hood. After drying, OC on the filter punch was measured by the thermal-optical OC/EC analyzer and the concentration in the extracts was calculated by dividing OC mass with pipetted extraction volume. Some extracted organic materials may evaporate with methanol during drying in the fume hood, which results in underestimation of OC content. The organic materials which may co-evaporate should have very high volatility. Since the filters were heated at $170^{\circ} \mathrm{C}$ to remove SVOC (including the organic materials with high volatility), the amount of organic materials which co-evaporate should be negligible. In order to determine fraction of total OC extracted by methanol, OC content on the quartz filter after methanol extraction was also analyzed by OC/EC analyzer. OC loading for methane residual on the filter after drying was negligible compared with OC samples.

\subsubsection{Semi-volatile organic carbon (SVOC)}

In the sampling gas, fresh OC concentrations were a few orders of magnitude higher than that diluted to ambient condition, which leads to a substantial part of SVOC partition to the particle phase. Some of the SVOC could be in the gaseous phase under ambient condition (Donahue, et al., 2006). In order to investigate mass and absorption of SVOC and non-volatile organic carbon (NVOC) 

separately, we removed SVOC in low flow rate samples by heating the sampled quartz filters at $212170^{\circ} \mathrm{C}$ at the helium mode in the $\mathrm{OC} / \mathrm{EC}$ analyzer. This temperature roughly reproduced atmospheric 213 partitioning but did not cause charring of OC. This method was previously used by Chen and Bond 214 (2010). We analyzed OC concentration and its absorbance in methanol extract for both original 215 filters (representing total organic carbon, TOC) and heated filter (representing NVOC). The 216 difference between those two is the contribution of SVOC.

$217 \quad 2.3$ Data analysis

218 The main values reported in this paper are absorption per mass of organic aerosol $(\alpha / \rho)$, 219 imaginary refractive index of organic aerosol $\left(k_{O A}\right)$ and absorption Ångström exponent (AAE). We 220 describe how these values are deduced as follows:

$221 \quad 2.3 .1$ Absorption per mass compound density in the unit of $\mathrm{m}^{2} / \mathrm{g}$. Here $\alpha$ is the absorption coefficient in liquid, and $\rho$ is the 224 density of dissolved organic compounds. The $\alpha / \rho$ values can be derived from Equation 1.

$225 \frac{\alpha}{\rho}(\lambda)=\frac{A(\lambda)}{c \times L} \times \ln (10)$

226 Where $A$ is the absorbance value measured by the spectrophotometer at the wavelength $\lambda, L$ is the 227 optical path length and equal to $1 \mathrm{~cm}$, and $c$ is the concentration of the organic carbon in the extract 228 determined by the OC/EC analysis as described in 2.2.3. A similar approach has been used by Chen 229 and Bond (2010). The $\alpha / \rho$ has the same unit $\left(\mathrm{m}^{2} / \mathrm{g}\right)$ as MAC, which is a fundamental input to 230 radiative transfer models. However, the two describe different properties and should not be confused 231 The $\alpha / \rho$ in our study is bulk absorption by liquid extract, however, MAC is value of absorption by 232 particles. MAC can be compared with $\alpha / \rho$ only after considering the particulate effect, $\xi$ (Sun et al., 233 2007). 
$234 \quad M A C=\frac{\alpha}{\rho} \varepsilon$

235 Where $\xi$ is a function of $n$ and $k$ :

$236 \quad \varepsilon=\frac{9 n}{\left(n^{2}-k^{2}+2\right)^{2}+4 n^{2} k^{2}}$

$237 \quad$ 2.3.2 Imaginary refractive index $\left(k_{O A}\right)$

238 The complex refractive index $(m=n-i k)$ is the fundamental parameter in Mie theory to calculate 239 mass scattering and absorption cross section (MSC and MAC). The values $n$ and $k$, i.e., real and 240 imaginary part of the complex refractive index, are mainly associated with the scattering and 241 absorption, respectively. This paper focuses on absorption, so we report values of $k$. The $k_{O A}$ can be 242 derived from Equation 4.

$243 \alpha=4 \pi k / \lambda$

244 Here $\alpha$ was calculated from the determined $\alpha / \rho$ values with Equation 1 by assuming an organic 245 compound density ( $\rho$ ) of 1.2, which is a commonly used density for OC (Turpin and Lim, 2001).

246 2.3.3 Absorption Ångström exponent

Absorption Ångström exponent (AAE), a parameter used to describe the spectral dependence of aerosol light absorption, is defined as:

$$
A A E=\frac{-\ln \left(\frac{\alpha\left(\lambda_{1}\right)}{\alpha\left(\lambda_{2}\right)}\right)}{\ln \left(\frac{\lambda_{1}}{\lambda_{2}}\right)}
$$

250 The AAE values were determined by means of linear regression of $\ln (\alpha / \rho)$ versus $\ln (\lambda)$.

251 3. Results and discussion

2523.1 Fraction of total OC extracted by methanol

253 The fraction of OC extracted by methanol at three pyrolysis temperatures ranged from $90.6 \%$ to $25498.2 \%$. Average fractions are $95.3 \pm 1.8 \%, 96.1 \pm 1.4 \%$ and $93.7 \pm 2.3 \%$ at $210{ }^{\circ} \mathrm{C}, 270{ }^{\circ} \mathrm{C}$ and $360{ }^{\circ} \mathrm{C}$, 
respectively. Our results for aerosol from pyrolysis of corn stalk are consistent with previous results

256 from wood pyrolysis (Chen and Bond, 2010). The high extraction fraction by methanol indicated that

257 absorption by methanol extraction could represent the behavior of the total OC. No obvious

258 difference was found in OC extraction fraction by methanol at different pyrolysis temperatures.

$259 \quad 3.2$ Effect of organic vapors adsorbed on the quartz filters

Adsorbed organic vapors on the backup quartz filters contributes only a small fraction to the total OC mass. Its average percentages are $4.0 \%, 4.7 \%$ and $7.5 \%$ at $210{ }^{\circ} \mathrm{C}, 270{ }^{\circ} \mathrm{C}, 360{ }^{\circ} \mathrm{C}$ pyrolysis temperatures, respectively. Its contribution to total OC increases with pyrolysis temperature 263 increasing.

Absorbance values of extracts from adsorbed organic vapors are much less than those from low flow rate branch (representing TOC). Even in the ultraviolet region, the values are lower than the instrument detection limit.

SVOC, determined by heating samples to $170^{\circ} \mathrm{C}$, contributes significantly to the total OC mass, with average percentages of $51.1 \%, 52.8 \%$ and $52.7 \%$ at $210{ }^{\circ} \mathrm{C}, 270{ }^{\circ} \mathrm{C}, 360{ }^{\circ} \mathrm{C}$ pyrolysis temperatures, respectively. When heated at $170^{\circ} \mathrm{C}$, absorbed organic vapors are also released, so SVOC in our study also includes adsorbed organic vapors.

The curves in Figure 3 indicate the ratio of absorbance by NVOC over that by TOC for samples 273 generated at different temperatures. NVOC contributes $56.8 \%, 58.4 \%$ and $65.8 \%$ of the absorbance 274 at $360 \mathrm{~nm}$ at $210{ }^{\circ} \mathrm{C}, 270{ }^{\circ} \mathrm{C}, 360^{\circ} \mathrm{C}$ pyrolysis temperatures, respectively. This indicates that the 275 removed SVOC does contribute to the light absorption in the ultraviolet region, and we need to 276 account for this effect when estimating the absorption of NVOC. Increased absorbances after 277 removal of SVOC are observed in the visible range, and it could have several causes. One is 278 chemical changes of organics when heated, and the other is measurement error because the measured absorbance values in the visible range were below the instrument detection limit. We have not 
conducted further study since OC absorption in the visible range is negligible, so we assume that

281 SVOC will not contribute to the light absorption in the visible range.

As discussed in Section 2.2.4, SVOC should be in the gaseous phase when organic aerosol generated by the pyrolysis process was diluted to ambient condition. To provide data suitable for radiative-transfer models, SVOC should be separated from NVOC. In the following section, we only provide data for optical properties for NVOC.

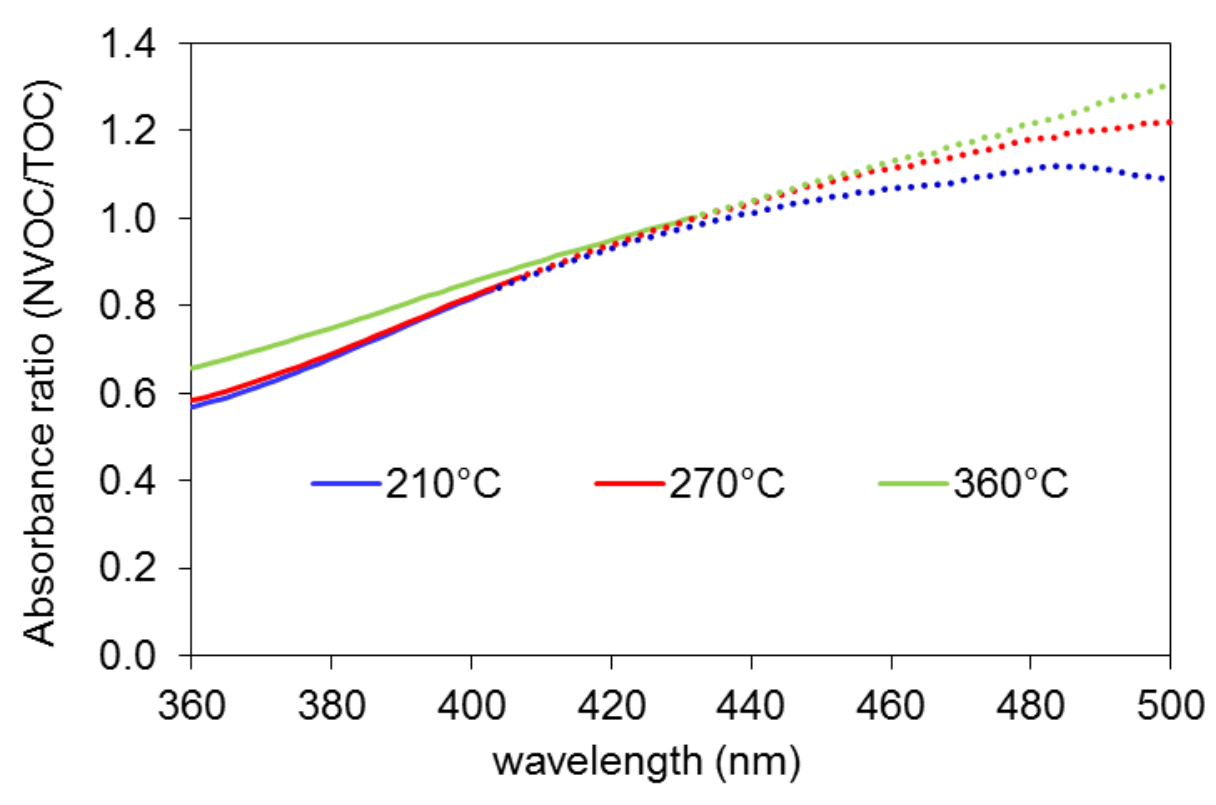

Fig.3 Ratio of absorbance of NVOC and TOC (Dashed lines indicates the measured absorbance values are blow the instrument detection limit).

In this study, we investigated three different pyrolysis temperatures: $210{ }^{\circ} \mathrm{C}, 270{ }^{\circ} \mathrm{C}, 360^{\circ} \mathrm{C}$. Actual temperature varied $10^{\circ} \mathrm{C}$ from the nominal temperature. Figure 4 shows average and standard deviation of absorption per mass of organic carbon $(\alpha / \rho)$ at three pyrolysis temperatures. It illustrates that $\alpha \rho$ increases with increasing pyrolysis temperatures across the investigated temperature range. An increase from $210{ }^{\circ} \mathrm{C}$ to $270{ }^{\circ} \mathrm{C}$ and $210{ }^{\circ} \mathrm{C}$ to $360{ }^{\circ} \mathrm{C}$ results in approximately $20 \%$ and $50 \%$ increase of $\alpha / \rho$ (average in ultraviolet range), respectively. Chen and Bond (2010) also observed the similar results for wood pyrolysis. High temperature favors polymerization of volatile matter before 
297 it exits the fuel, which results in greater absorption per mass. However, the degree of $\alpha / \rho$ increase with temperature is much lower than that of wood pyrolysis (Chen and Bond, 2010). Difference in $\alpha / \rho$ for wood pyrolysis at $210{ }^{\circ} \mathrm{C}$ and $360^{\circ} \mathrm{C}$ was observed for a few times. The discrepancy may be due to the difference in fuel structure. Corn stalk has a looser fiber structure relative to wood. During the pyrolysis of the corn stalk, OC precursors are released more easily, and hence have lesse residence time. This weakens the polymerization and results less increase of $\alpha / \rho$ than in the wood pyrolysis.

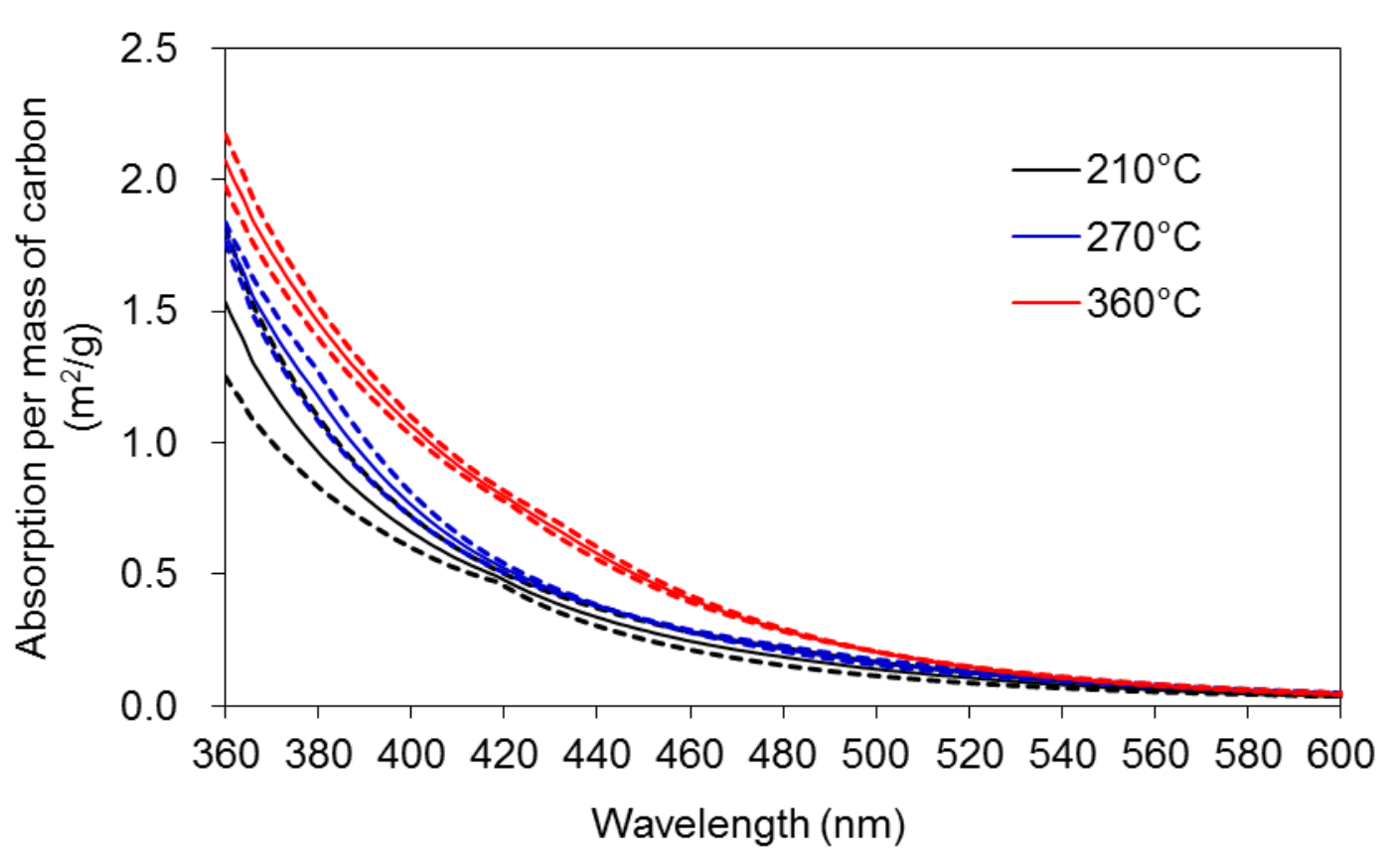

305 Fig.4 Average absorption per mass of organic carbon $(\alpha / \rho)$ at three pyrolysis temperatures. Dashed lines with the same color indicate upper and lower range.

We list $\alpha / \rho$ at different wavelengths from this study and along with similar values in our prior works in Table 1 . Values of $\alpha / \rho$ for corn stalk pyrolysis are comparable with those for wood pyrolysis. In general, values of $\alpha / \rho$ for corn stalk are higher than wood at $210^{\circ} \mathrm{C}$ and $270^{\circ} \mathrm{C}$ pyrolysis temperatures. However, the situation is the opposite at $360^{\circ} \mathrm{C}$ pyrolysis temperature, possibly because of greater polymerization in wood at this temperature. It may also relate to difference on

312 biomass constituents. Wood has a higher lignin content relative to corn stalk (McKendry, 2002). 
313 Lignin, an essential and major biopolymer of biomass tissue, is derived primarily from aromatics 314 alcohols (Simoneit, 2002). Pyrolyzed at $360^{\circ} \mathrm{C}$, a large amount of lignin decomposes (Collard and 315 Blin, 2014), polymerizes, and releases the aerosol enriched of aromatic moiety (Simoneit, 2002), 316 which is thought as high absorption (Chen and Bond, 2010).

317 Table 1. Average absorption per mass $(\alpha / \rho)$ at different wavelength from this study and literature 318

\begin{tabular}{|c|c|c|c|c|c|c|}
\hline \multirow{2}{*}{$\begin{array}{c}\lambda \\
(\mathrm{nm})\end{array}$} & \multicolumn{3}{|c|}{ corn stalk pyrolysis } & \multicolumn{3}{|c|}{ wood pyrolysis ${ }^{\mathrm{a}}$} \\
\hline & $210^{\circ} \mathrm{C}$ & $270^{\circ} \mathrm{C}$ & $360^{\circ} \mathrm{C}$ & $210^{\circ} \mathrm{C}$ & $270^{\circ} \mathrm{C}$ & $360^{\circ} \mathrm{C}$ \\
\hline 360 & 1.53 & 1.80 & 2.07 & 1.55 & 1.79 & 3.06 \\
\hline 400 & 0.66 & 0.76 & 1.06 & 0.50 & 0.68 & 1.66 \\
\hline 450 & 0.29 & 0.33 & 0.48 & 0.15 & 0.24 & 0.68 \\
\hline 500 & 0.14 & 0.16 & 0.20 & 0.07 & 0.10 & 0.27 \\
\hline 550 & 0.07 & 0.09 & 0.09 & 0.03 & 0.05 & 0.11 \\
\hline
\end{tabular}

321 Figure 5 shows the average and standard deviation of imaginary part of refractive index $(k)$ at 322 three pyrolysis temperatures. As with $\alpha / \rho, k_{O A}$ increases with increasing pyrolysis temperatures. 


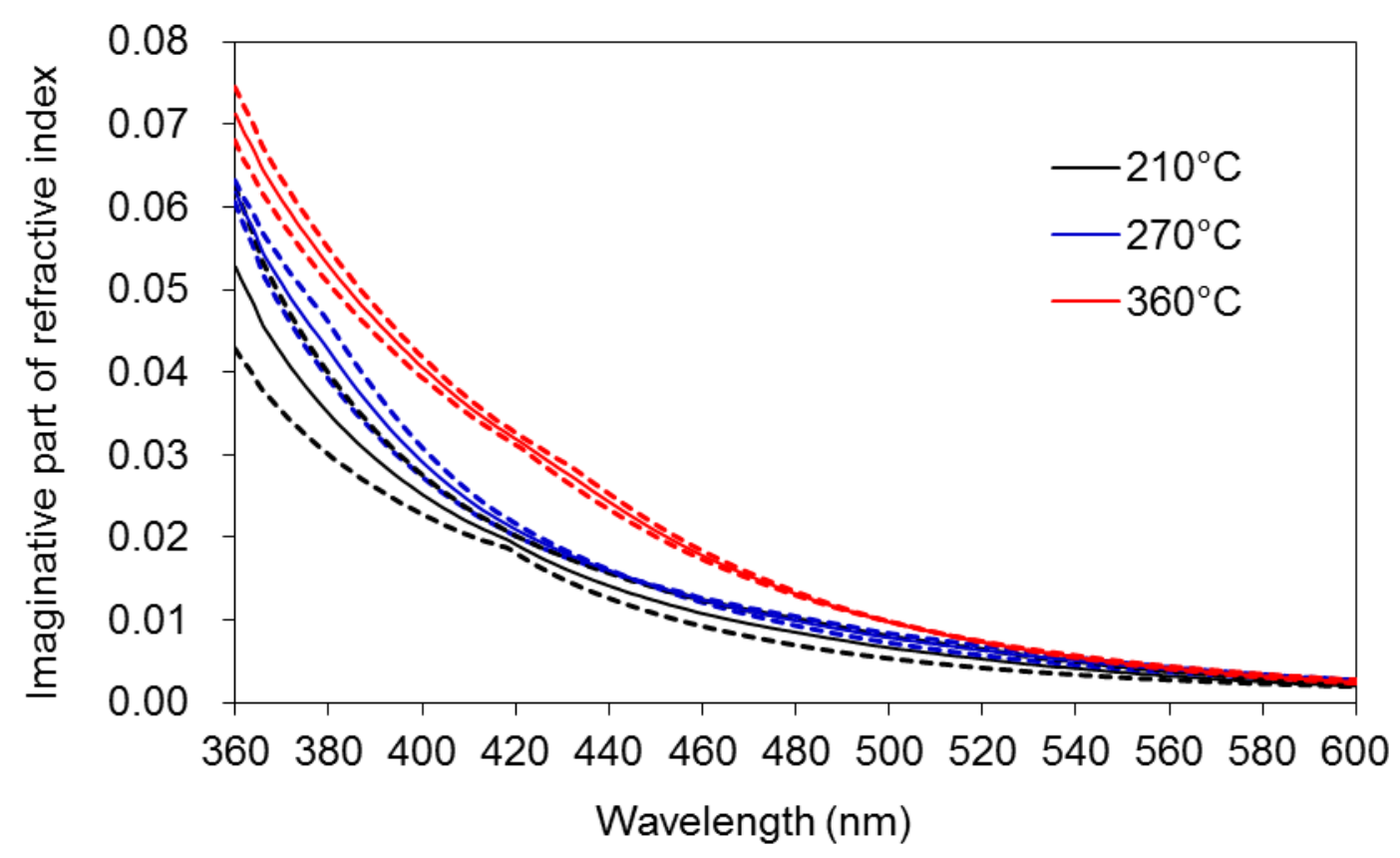

Fig.5 Average imaginary part of refractive index $\left(k_{O A}\right)$ at three pyrolysis temperatures. Dashed lines with the same color indicate upper and lower range. our $k_{O A}$ values for corn stalk pyrolysis are comparable with those for wood pyrolysis (Chen and Bond,

Since $k_{O A}$ is an intrinsic property, we now compare our $k_{O A}$ values with other values found in the literature. Table 2 shows a summary of $k_{O A}$ obtained using various methods for various types of biomass burning aerosols. We choose only $k$ values for primary organic aerosol (POA) from biomass burning excluding other emission sources, secondary organic aerosol (SOA) and HULIS. As with $\alpha / \rho$, 2010). However, our k values are a few times lower than those reported by Kirchstetter et al. (2004). Kirchstetter et al. (2004) used filter-based optical transmission method to determine difference in light transmission of the loaded filters before and after extraction with acetone (aerosols mixed with organic and black carbon), and then calculated optical constants including $k_{O A}$. They admitted this method may overestimated absorption due to multiple scattering by the filter medium. Other possible reasons for the discrepancy are particle light scattering effect, and liquid organic material coating the filter fiber (Bond et al., 1999; Subramanian et al., 2007).

Our $k_{O A}$ values are much higher than the two studies that used optical closure methods (Chakrabarty et al., 2010; Lack et al., 2012). Chakrabarty et al. (2010) used a photoacoustic 
spectrometer to determine scattering and absorption coefficients of particles emitted from smoldering combustion of two commonly occurring dry mid-latitude fuels, and then retrieved the complex refractive indices of the particles by adjusting the refractive index used as input in the Mie theory to match the measured scattering and absorption data. In their study, no detectable elemental carbon was found in the particles, so particle light absorption can be considered to be from organic carbon.

Lack et al. (2012) used multi-wavelength photoacoustic aerosol absorption spectrometer to measure absorption coefficients of mixed particles emitted from ponderosa pine forest fire, and applied Mie

Theory modelling to calculate $\mathrm{BC}$, internal mixing and $\mathrm{OC}$ contribution to the absorption, and retrieved $k_{O A}$ at $404 \mathrm{~nm}$. These values of $k_{O A}$ would be an average over all OC, including semivolatile material.

work indicates that almost all of the OA absorption was associated with extremely low-volatile organic compounds (ELVOCs). They obtained values of $k_{O A}$ using several assumptions (see table 
encouraged in the future. This effort will be helpful to explain the discrepancy among different

366 methods. Our improved extraction method extends OC absorption measurement to around 600nm in

367 this study, and longer wavelength could be achieved if more careful experiments are designed. The

368 improvement makes the comparison between extraction methods and the multi-wavelength in-situ

369 methods (such as PASS-3, a commercialized photoacoustic spectrometer, is operated at 405nm,

$370 \quad 532 \mathrm{~nm}$ and $781 \mathrm{~nm})$ possible.

371 Table 2. Summary of $\mathrm{k}$ and AAE of OC obtained using various methods for various types of biomass burning aerosols.

\begin{tabular}{|c|c|c|c|c|c|}
\hline Aerosol source & $\lambda(\mathrm{nm})$ & $k$ & AAE & Method & Reference \\
\hline Pyrolysis of & 400 & $0.041^{b}$ & 7.7 & Extract bulk light & This study \\
\hline corn stalk $^{\mathrm{a}}$ & 550 & 0.005 & & absorbance measurement & \\
\hline Pyrolysis of & 400 & $0.063^{b}$ & $6.9-7.8$ & Same as before & Chen and Bond, \\
\hline wood $^{\mathrm{a}}$ & 550 & 0.006 & & & 2010 \\
\hline Firewood smoke & 400 & 0.11 & $1.2-2.2^{\mathrm{d}}$ & Filter-based optical & Kirchstetter et al., \\
\hline and savanna fires ${ }^{c}$ & 550 & 0.03 & & transmission measurement $\mathrm{e}^{\mathrm{e}}$ & 2004 \\
\hline Smoldering biomass & 405 & $0.0076 \sim 0.015$ & $4.2 \sim 6.4^{\mathrm{f}}$ & Optical closure $^{\mathrm{g}}$ & Chakrabarty et al., \\
\hline combustion $^{\mathrm{a}}$ & 532 & $0.002 \sim 0.008$ & $2.3 \sim 2.4$ & & 2010 \\
\hline Ponderosa pine & 404 & 0.009 & $2.3^{\mathrm{d}}$ & Optical closure $^{\mathrm{g}}$ & Lack et al., 2012 \\
\hline forest fire $^{c}$ & & & & & \\
\hline \multirow[t]{2}{*}{ Biomass burning $^{c}$} & 550 & $0.0055 \sim 0.02^{\mathrm{h}}$ & $3.2 \sim 3.9^{h}$ & Optical closure $^{\mathrm{g}}$ & Saleh et al., 2013 \\
\hline & & $0.022 \sim 0.06^{\mathrm{i}}$ & $3.1 \sim 4.2^{\mathrm{i}}$ & & \\
\hline \multirow[t]{4}{*}{ Biomass burning $^{c}$} & 550 & $0.006 \sim 0.035^{\mathrm{j}}$ & $1.5 \sim 5.4^{\mathrm{j}}$ & Optical closure $^{g}$ & Saleh et al., 2014 \\
\hline & & $0.007 \sim 0.037^{\mathrm{k}}$ & $1.4 \sim 5.3^{\mathrm{k}}$ & & \\
\hline & & $0.07 \sim 0.22^{1}$ & $2.1 \sim 2.4^{1}$ & & \\
\hline & & $0.09 \sim 0.24^{\mathrm{m}}$ & $1.9 \sim 2^{\mathrm{m}}$ & & \\
\hline
\end{tabular}


$373 \quad{ }^{a}$ Organic carbon, no black carbon.

$374{ }^{\mathrm{b}}$ Values at pyrolysis temperature $360^{\circ} \mathrm{C}$.

$375{ }^{\mathrm{c}}$ Mixed aerosol, including OC and BC.

$376{ }^{\mathrm{d}}$ Values for mixed aerosol

$377{ }^{\mathrm{e}}$ Measure difference in light transmission of the loaded filters before and after extraction with 378 acetone.

$379 \mathrm{f}$ AAE values at 4.2-6.4 are for wavelength between 405 and 532nm; those at 2.3-2.4 are for 380 wavelength between 532-780nm.

381 "The complex refractive indices of the particles were retrieved from Mie "closure" calculations 382 based on particle size, extinction and absorption measurements.

$383{ }^{\mathrm{h}}$ Assuming BC and OA are externally-mixed.

$384{ }^{\mathrm{i}}$ Assuming BC are internally-mixed with OA.

$385{ }^{\mathrm{j}}$ Assuming BC are internally-mixed with total OA.

$386{ }^{\mathrm{k}}$ Assuming BC and OA are externally-mixed with total OA.

$387{ }^{1}$ Assuming BC are internally-mixed with ELVOCs.

$388{ }^{\mathrm{m}}$ Assuming BC and OA are externally-mixed with ELVOCs.

389 3.6 Absorption Ångström exponent (AAE)

$390 \quad$ Values of AAE for OC extracted by methanol at three pyrolysis temperatures are shown in 391 Table 2. The values range from 7.0 to 7.7 in the whole investigated range, showing strong spectral 392 dependence. Those are similar to the data reported by Chen and Bond (2010) for organic carbon from 393 wood pyrolysis (6.9-11.4), but much higher than the values derived from aerosols mixed with 394 organic and black carbon from biomass burning emissions (1.2-5.4) (Saleh et al., 2013; 2014; 395 Kirchstetter et al., 2004; Lack et al., 2012), and also higher than those of OC from smoldering 396 biomass burning (Chakrabarty et al., 2010).

397 4. Summary and conclusions 
With the improved the extraction method and extended the spectral range of OC absorption, we investigated light absorption of organic aerosol from pyrolysis of corn stalks at different temperatures. The absorption shows strong spectral dependence $(\mathrm{AAE}=7.7)$ and is not be negligible at shorter wavelengths. Absorption per mass of organic carbon increases with increasing pyrolysis temperatures in the range of temperatures we investigated $\left(210{ }^{\circ} \mathrm{C}\right.$ to $\left.360{ }^{\circ} \mathrm{C}\right)$. Imaginary refractive index of organic aerosol $\left(k_{O A}\right)$ were determined and compared with other values found in the literature.

Measured absorption in extracted OA appears to have some consistency, regardless of whether the OA is generated from wood or from agricultural waste, although the effect of pyrolysis temperature depends on the biomass type. We confirm the finding that semi-volatile organic carbon adsorbs primarily at ultraviolet wavelengths, and including it in measurements reduces the apparent imaginary refractive index at visible wavelengths. However, imaginary refractive index values inferred from optical closure depend upon a few assumptions about particle properties.

In order to gain a more fundamental understanding of factors affecting the absorptive properties of isolated organic aerosol and aerosol mixed with other compounds, an inter-comparison study of different methods to determine light absorbing properties of OC should be conducted in the future.

414 This effort will be helpful to explain the discrepancy among different methods.

\section{Acknowledgements}

This work is supported by the U.S. DOE research grant: "Isolating Weakly and StronglyAbsorbing Classes of Carbonaceous Aerosol: Optical Properties, Abundance and Wet Removal" (Grant No.A0871 DOE DE-SC0006689). Xinghua Li was partly supported by National Natural 420 Science Foundation of China (Grant No. 41275121 and 41575119) and Chinese National Foundation 421 for Studying Abroad (China Scholarship Council). 
424 Alexander, D. L. T., P. A. Crozier, and J. R. Anderson (2008), Brown carbon spheres in East Asian 425 outflow and their optical properties, Science, 321, 833-836.

426 Andreae, M. O., and A. Gelencser (2006), Black carbon or brown carbon? The nature of light427 absorbing carbonaceous aerosols, Atmos. Phys. Chem., 6, 3131-3148, doi:10.5194/acp-6-3131-2006.

428 Bond, T. C., T. L. Anderson, and D. Campbell (1999), Calibration and intercomparison of filter429 based measurements of visible light absorption by aerosols, Aerosol Sci. Technol., 30, 582-600.

430 Bond, T. C., D. G. Streets, K. F. Yarber, S. M. Nelson, J.- H. Woo, and Z. Klimont (2004), A 431 technology-based global inventory of black and organic carbon emissions from combustion, $J$. 432 Geophys. Res., 109, D14203, doi:10.1029/2003JD003697.

433 Brem, B. T., F. C. M. Gonzalez, S. R. Meyers, T. C. Bond, and M. J. Rood (2012), Laboratory434 measured optical properties of inorganic and organic aerosols at relative humidities up to $95 \%$, 435 Aerosol Sci. Tech., 46(2), 178-190, doi: 10.1080/02786826.2011.617794.

436 Cao, G., X. Zhang, F. Zheng (2006), Inventory of black carbon and organic carbon emissions from 437 China, Atmos. Environ., 4, 6516-6527.

438 Chakrabarty, R. K., H. Moosmuller, L. W. A. Chen, K. Lewis, W. P. Arnott, C. Mazzoleni, M. K. 439 Dubey, C. E. Wold, W. M. Hao, and S. M. Kreidenweis (2010), Brown carbon in tar balls from 440 smoldering biomass combustion, Atmos. Chem. Phys., 10(13), 6363-6370, doi: 10.5194/acp-10$441 \quad 6363-2010$.

442 Chen, Y., and T. C. Bond (2010), Light absorption by organic carbon from wood combustion, Atmos. 443 Chem. Phys., 10(4), 1773-1787. 
444 Chen, Y., C. A. Roden, and T. C. Bond (2012), Characterizing biofuel combustion with patterns of 445 real-time emission data (PaRTED), Environ. Sci. Technol. 46, 6110-6117.

446 Chow, J. C., J. G. Watson, L. -W. A. Chen, W. P. Arnott, and H. Moosmüller (2004), Equivalence of 447 Elemental Carbon by Thermal/Optical Reflectance and Transmittance with Different Temperature 448 Protocols, Environ. Sci. Technol. 38, 4414-4422.

449 Chung, S. H., and J. H. Seinfeld (2002), Global distribution and climate forcing of carbonaceous 450 aerosols, J. Geophys. Res.,107, 4407, 10.1029/2001jd001397.

451 Collard, F.-X., J. Blin (2014), A review on pyrolysis of biomass constituents: Mechanisms and 452 composition of the products obtained from the conversion of cellulose, hemicelluloses and lignin, 453 Renewable and Sustainable Energy Reviews, 38, 594-608.

454 Donahue, N. M., A. L. Robinson, C. O. Stanier, and S. N. Pandis (2006), Coupled partitioning, 455 dilution, and chemical aging of semivolatile organics, Environ. Sci. Technol., 40, 2635-2643.

456 Einfeld, W., D. E. Ward, and C. Hardy (1991), Effects of fire behavior on prescribed fire smoke 457 characteristics: A case study, in: Global biomass burning: Atmospheric, climatic, and biospheric 458 implications, MIT Press, Cambridge, MA, USA, 412-419.

459 Favez, O., S. C. Alfaro, J. Sciare, H. Cachier, M. M. Abdelwahab (2009), Ambient measurements of 460 light-absorption by agricultural waste burning organic aerosols, J. Aerosol Sci., 40, 613-620.

461 Feng, Y., V. Ramanathan, V. R. Kotamarthi (2013), Brown carbon: A significant atmospheric 462 absorber of solar radiation? Atmos. Chem. Phys., 13, 8607-8621.

463 Kanakidou M., J. H. Seinfeld, S. N. Pandis, et al. (2005), Organic aerosol and global climate 464 modelling: A review, Atmos. Chem. Phys., 5, 1053-1123. 
465 Kirchstetter, T. W., T. Novakov, and P. V. Hobbs (2004), Evidence that the spectral dependence of 466 light absorption by aerosols is affected by organic carbon, J. Geophys. Res., 109, D21208, 467 doi:10.1029/2004JD004999.

468 Lack, D. A., J. M. Langridge, R. Bahreini, C. A. Brock, A. M. Middlebrook, and J. P. Schwarz 469 (2012), Brown carbon and internal mixing in biomass burning particles, P. Natl. Acad. Sci., 109, 470 14802-14807, doi:10.1073/pnas.1206575109.

471 Li, X., S. Wang, L. Duan, J. Hao, Y. Nie (2009), Carbonaceous aerosol emissions from household 472 biofuel combustion in China, Environ. Sci. Technol., 43: 6076-6081.

473 Li, X., S. Wang, L. Duan, J. Hao, C. Li, Y. Chen, L. Yang (2007), Particulate and Trace Gas

474 Emissions from Open Burning of Wheat Straw and Corn Stover in China, Environ. Sci. Technol., 41, $475 \quad 6052-6058$.

476 Lin, G. X., J. E. Penner, M. G. Flanner et al. (2014), Radiative forcing of organic aerosol in the 477 atmosphere and on snow: Effects of SOA and brown carbon, J. Geophys. Res., 119(12), $7453-$ 478 7476, doi: 10.1002/2013JD021186.

479 Lu, Z., D. G. Streets, E. Winijkul, et al. (2015), Light Absorption Properties and Radiative Eff ects of 480 Primary Organic Aerosol Emissions, Environ. Sci. Technol., 49, 4868-4877.

481 McKendry, P. (2002), Energy production from biomass (part 1): Overview of biomass, Bioresour 482 Technol., 83, 37-46.

483 Ming, Y., V. Ramaswamy, P. A. Ginoux, and L. H. Horowitz (2005), Direct radiative forcing of 484 anthropogenic organic aerosol, J. Geophys. Res.-Atmos, 110, D20208, 10.1029/2004jd005573, 2005.

485 Myhre, G., N. Bellouin, T. F. Berglen, T. K. Berntsen, O. Boucher, A. Grini, I. S. A. Isaksen, M. 486 Johnsrud, M. I. Mishchenko, F. Stordal, and D. Tanre (2007), Comparison of the radiative properties 487 and direct radiative effect of aerosols from a global aerosol model and remote sensing data over 
ocean, Tellus Series B-Chemical and Physical Meteorology, 59, 115-129, 10.1111/j.16000889.2006.00226.x, 2007.

NIOSH (2003), Manual of analytical methods 5040, issue 3, edited, National Institute for Occupational Safety and Health.

Park, R. J., M. J. Kim, J. I. Jeong, D. Youn, S. Kim (2010), A contribution of brown carbon aerosol to the aerosol light absorption and its radiative forcing in East Asia, Atmos. Environ., 44, 1414-1421.

Roden C. A., T. C. Bond, S. Conway, B. A. O. Pinel (2006), Emission factors and real-time optical properties of particles emitted from traditional wood burning cookstoves, Environ. Sci. Technol., 40, 6750-6757.

Saleh, R., C. J. Hennigan, G. R. McMeeking, W. K. Chuang, E. S. Robinson, H. Coe, N. M.

Donahue and A. L. Robinson (2013), Absorptivity of brown carbon in fresh and photo-chemically aged biomass-burning emissions, Atmos. Chem. Phys., 13, 7683-7693.

Saleh, R., E. S. Robinson, D. S. Tkacik, A. T. Ahern, S. Liu, A. C. Aiken, R. C. Sullivan, A. A. Presto, M. K. Dubey, R. J. Yokelson, N. M. Donahue, A. L. Robinson (2014), Brownness of organics in aerosols from biomass burning linked to their black carbon content, Nat. Geosci., 7, 647-650.

Simoneit, B. R.T. (2002), Biomass burning-a review of organic tracers for smoke from incomplete combustion, Applied Geochemistry, 17, 129-162.

Subramanian, R., C. A. Roden, P. Boparai, and T. C. Bond (2007), Yellow beads and missing particles: Trouble ahead for filter-based absorption measurements, Aerosol Sci. Technol., 41, 630$637,10.1080 / 02786820701344589$.

Sun, H. L., L. Biedermann, and T. C. Bond (2007), Color of brown carbon: A model for ultraviolet and visible light absorption by organic carbon aerosol, Geophys. Res. Lett., 34, L17813, $10.1029 / 2007 \mathrm{~g} 1029797$. 
511 Turpin, B. J., J. J. Huntzicker, and S. V. Hering (1994), Investigation of organic aerosol sampling

512 artifacts in the Los-Angeles Basin, Atmos. Environ., 28, 3061-3071.

513 Turpin, B. J., and H. J. Lim (2001), Species contributions to PM2.5 mass concentrations: Revisiting 514 common assumptions for estimating organic mass, Aerosol Sci. Tech., 35(1), 602-610, doi:

$515 \quad 10.1080 / 02786820152051454$.

516 Yang, M., S. G. Howell, J. Zhuang, B. J. Huebert (2009), Attribution of aerosol light absorption to 517 black carbon, brown carbon, and dust in China interpretations of atmospheric measurements during 518 EASTAIRE, Atmos. Chem. Phys., 9, 2035-2050.

519 Zhang, Q., J. L. Jimenez, M. R. Canagaratna (2007), Ubiquity and dominance of oxygenated species 520 in organic aerosols in anthropogenically-influenced Northern Hemisphere midlatitudes, Geophys. 521 Res. Lett., 34, L13801, doi:10.1029/2007GL029979.

522 White, R., and M. Dietenberger (2001), Wood products: Thermal degradation and fire, Encyclopedia 523 of materials: science and technology.[S1]: Elsevier Science Ltd, 9712-9716. 
525 Graphical abstract

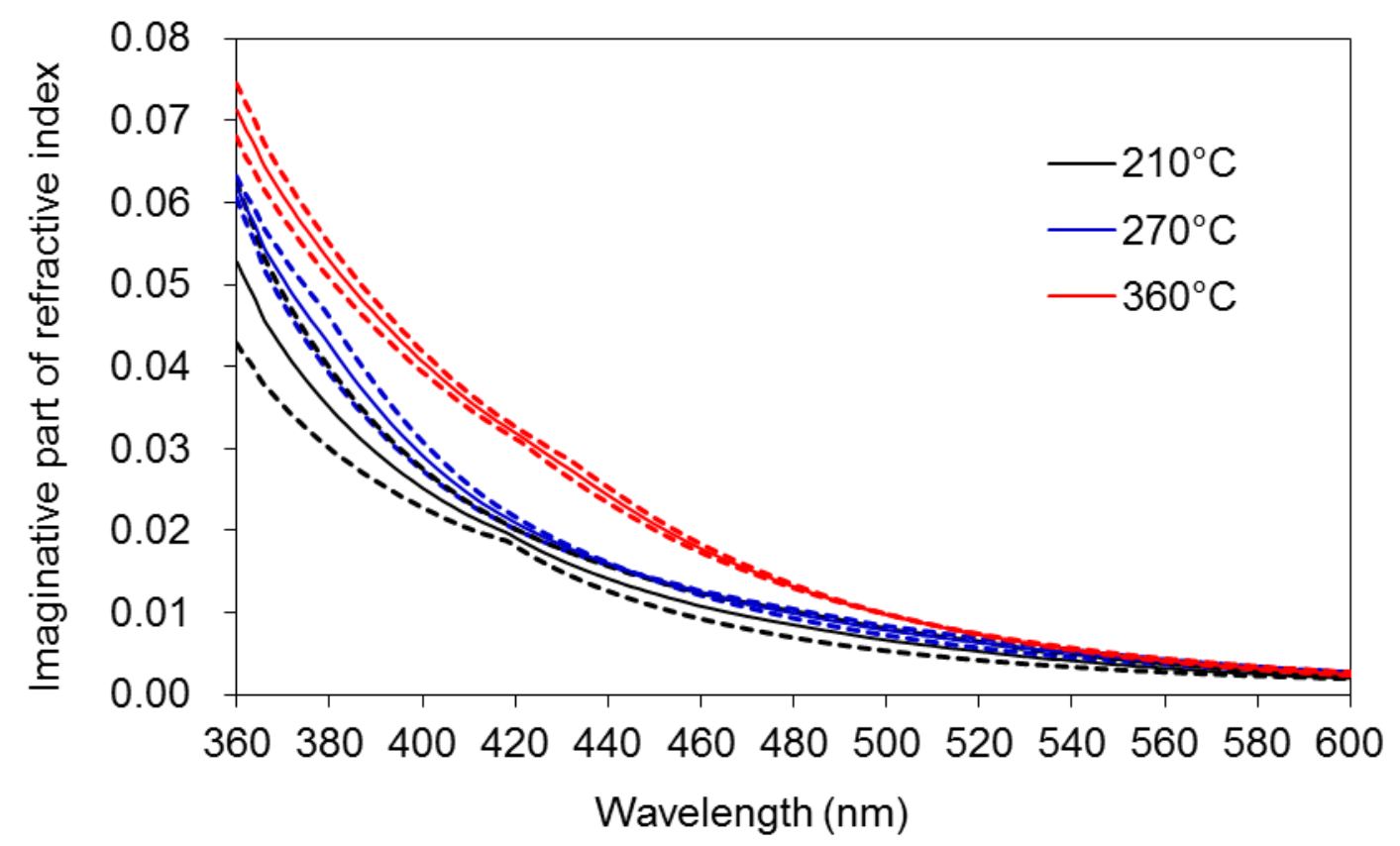

